\title{
Chemical Biology Approaches to Membrane Homeostasis and Function
}

\author{
Miwa Takahashi-Umebayashia, Ludovic Pineaua, Thomas Hannichª, Andreas Zumbuehla ${ }^{a}$, David \\ Alonso Doval ${ }^{a}$, Stefan Matilea, Christian Heinis ${ }^{b}$, Gerardo Turcatti ${ }^{b}$, Robbie Loewith ${ }^{a}$, Aurélien Roux ${ }^{b}$, \\ Luc Reymond ${ }^{b}$, Kai Johnsson ${ }^{b}$, and Howard Riezman*a
}

\begin{abstract}
The study of membranes is at a turning point. New theories about membrane structure and function have recently been proposed, however, new technologies, combining chemical, physical, and biochemical approaches are necessary to test these hypotheses. In particular, the NCCR in chemical biology aims to visualize and characterize membrane microdomains and determine their function during hormone signaling.
\end{abstract}

Keywords: Chemical biology · Fluorescent dyes · Giant unilamellar vesicles · Insulin receptor · Membranes · Microdomains $\cdot$ Rafts $\cdot$ Signaling $\cdot$ Sphingolipids $\cdot$ Sterols

\section{Introduction}

Membranes are essential components of cells that function to compartmentalize cellular events, control communication between compartments and with the exterior, allow the formation of gradients of ions and other solutes, and provide a proper environment for the large percentage of cellular proteins that are membrane bound or associated. Until recently, the role of lipids in the membrane bilayer was viewed as a rather boring support that blocks passage of proteins, nucleic acids and solutes, and dissolves the hydrophobic portion of membrane proteins. Even though we were aware of the great diversity of structures of membrane lipids and that membrane proteins and lipids are asymmetrically distributed in lipid bilayers, early models presented the membrane as a fluid-mosaic model, where membrane proteins diffuse freely in a homogeneous lipid environment. More recently, due to the convergence of findings that sphingolipids and sterols are co-enriched in some

\footnotetext{
${ }^{\star}$ Correspondence: Prof. Dr. H. Riezmana E-mail: Howard.Riezman@unige.ch aDepartments of Biochemistry, Organic Chemistry and Molecular Biology

University of Geneva

30 quai Ernest Ansermet

$\mathrm{CH}-1211$ Geneva 4

bInstitute of Chemical Sciences and Engineering Life Sciences

École Polytechnique Fédérale de Lausanne $\mathrm{CH}-1015$ Lausanne
}

cell membranes and that they can form liquid ordered domains in artificial lipid bilayers, a new hypothesis has emerged, termed the raft hypothesis.. ${ }^{1]}$ This new concept predicts that there are lateral heterogeneities in membranes that differ in their physical properties, especially with respect to the order parameter of the membrane. The hypothesis goes further in predicting that this order parameter plays an important role in many physiological events, including protein sorting and recruitment, signal transduction and membrane protein activity. One of the novelties of the raft hypothesis is that it postulates a role for specific lipids, in particular sphingolipids, sterols and glycerophospholipids with fully saturated acyl chains, in driving membrane phase separation/domain formation. The raft hypothesis is still far from proven even though physicochemical and molecular dynamic studies have shown that lipids can drive membrane domain formation in pure lipid systems. ${ }^{[2]}$ One of the major reasons for skepticism is the difficulty to visualize these domains in living cells. Under natural conditions domains or aggregates have been visualized, but they are very small with a diameter of around $50 \mathrm{~nm} \cdot{ }^{[3]}$ It has not been possible to colocalize proteins and lipids in membrane domains. Furthermore, maintenance of these domains seems to require energy and the actin cytoskeleton, which is difficult to reconcile with a lipid-driven phase separation. ${ }^{[4]}$ The only connection between these small protein domains and lipids is that they are disturbed by depletion of cholesterol. ${ }^{[5]}$ However, the consequences of ATP depletion and actin cytoskeleton disruption are different for lipids and proteins. The most likely explanation for this is that the lipids do not drive the formation of the protein domains.
On the other hand, attempts to visualize raft structures have led to the finding that membranes are not homogeneous. Single molecule tracking of proteins and lipids have shown that both molecules show a particular form of movement, termed hop diffusion. ${ }^{[6]}$ Both transmembrane proteins and lipids freely diffuse within restricted areas, over whose borders they skip. Again, this membrane organization seems to be affected by the underlying cortical actin cytoskeleton. High resolution microscopy using fluorescence correlation with fluorescently labeled lipids has also shown that lipid diffusion is restricted in certain domains of the plasma membrane. ${ }^{[7]}$ Again, cholesterol depletion seems to affect this organization. Another hypothesis that is consistent with the findings cited above is the lipid shell hypothesis. ${ }^{[8]}$ This concept states that membrane proteins are surrounded by a lipid shell coating their transmembrane domains. One of the major differences with the raft hypothesis is that the lipid shell hypothesis predicts that proteins organize the lipids rather than the inverse. Molecular dynamics experiments combining transmembrane peptides and lipids are consistent with the lipid shell hypothesis, but also relate it to the raft hypothesis. ${ }^{[9]}$ These studies suggest that proteins can serve to organize lipids around their transmembrane domains and that the sterols and sphingolipids associated with transmembrane domains can further organize more lipids.

One of the greatest difficulties in addressing membrane structure and domain organizations is the lack of techniques to visualize and modulate membrane domains and their properties. The NCCR Chemical Biology will develop new tools to visualize membrane lipids, membrane properties and interfere with lipid homeostasis. 


\section{Visualizing Sphingolipids}

Nature has already succeeded in creating peptides that recognize membrane lipids. Some notorious examples are the bacterial cholera and shiga toxins, which recognize glycolipids ${ }^{[10]}$ and the earthworm toxin lysenin that recognizes sphingomyelin. ${ }^{[11]}$ We will take advantage of the fact that peptides can be evolved to specifically bind sphingolipids together with the novel technology developed by a member of our NCCR using evolution of bicyclic peptides selected after phage display. ${ }^{[12]}$ To do this novel selection techniques will need to be developed that are compatible with lipid display. We will use yeast sphingolipids as a test system. Yeast sphingolipid biosynthesis is similarly organized as in higher eukaryotes, but yeast makes only inositol containing sphingolipids (Fig. 1). There are three major configurations in the sphingolipid head group, which are likely to be recognized, inositol phosphate, mannosylinositiol phosphate, and mannosyl diinositolphosphate. We will purify the three major classes of yeast sphingolipids and raise bicyclic peptides that recognize them. Once obtained there could be many uses for the peptides. They can be used to localize the sphingolipids in yeast cells by fluorescence microscopy or eventually by electron microscopy. The visualization can be combined with genetic techniques to identify the genes required for sphingolipid trafficking. The peptides can also be linked to toxic substances and used in genetic selections for yeast mutants that do not synthesize or transport these sphingolipids to the cell surface. Once this technology is developed it can be combined with isolation techniques or chemical synthesis to raise bicyclic peptides against other sphingoand glycolipids.

\section{Why Chemical Interference?}

The fundamental goal of biology is to understand the molecular function of biological factors and how they work in concert to produce the life forms that we know. These factors range from small molecule metabolites, to lipids, to large biopolymers such as sugar polymers, proteins or nucleic acids. Arguably, one of the best means to interrogate the function of biological factors is to observe the consequence their removal/inactivation has upon the system being studied. Presently, there are several approaches by which the activities and/or abundance of such factors can be targeted. In general terms, these approaches range from purely genetic to purely chemical; each approach has particular advantages and disadvantages. ${ }^{[13]}$

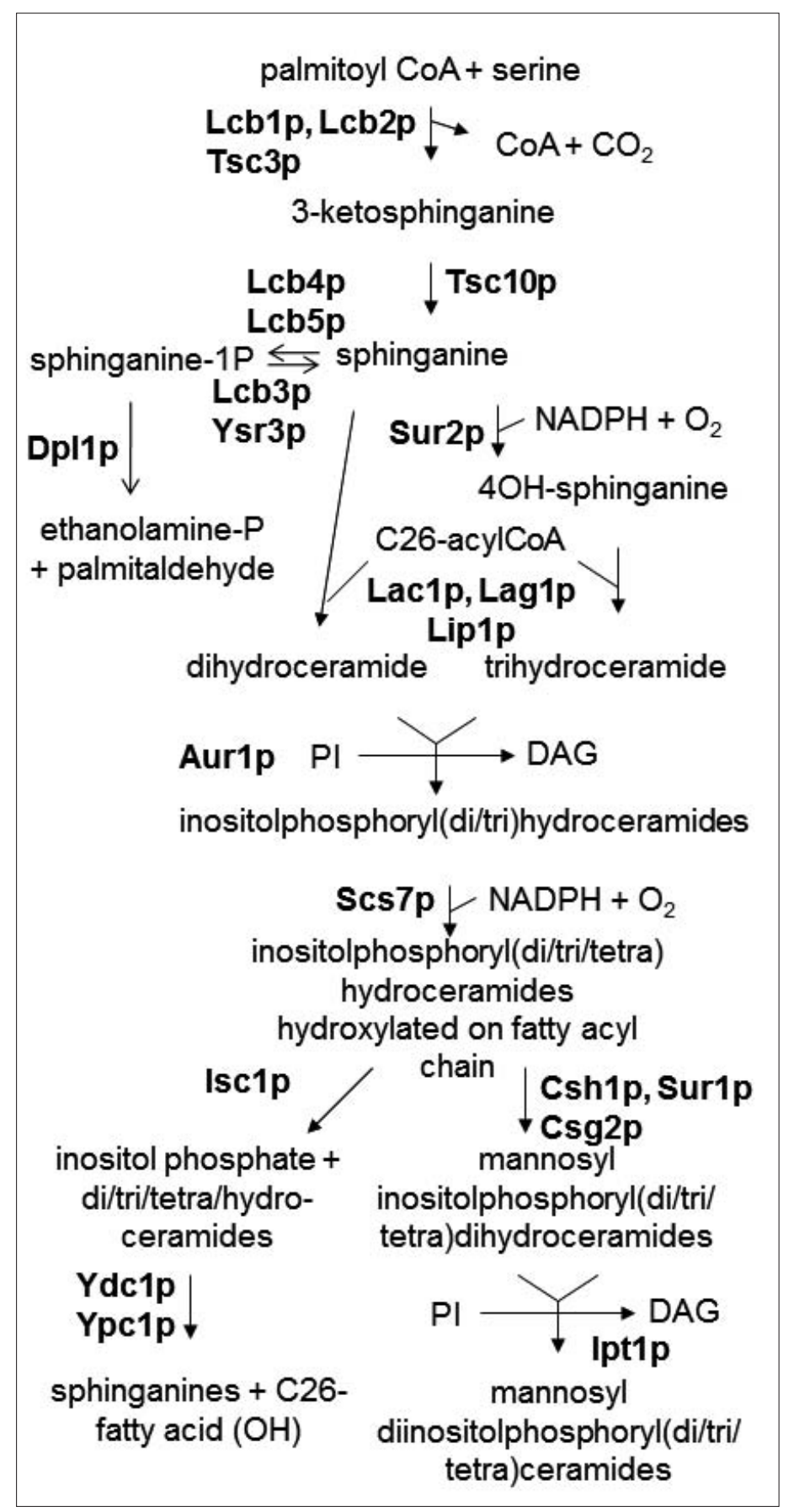

Fig. 1. Sphingolipid metabolism in yeast. The sphingolipid lipid biosynthetic and degradation pathways are wellcharacterized in the yeast Saccharomyces cerevisiae, and the major steps are shown here. The proteins corresponding to the enzymatic steps are shown in standard nomenclature. Lag1p and Lac1p are essential, redundant subunits of the yeast ceramide synthase and are deleted in the approach described in the text.
Traditionally, classical genetics has been the tool of choice to manipulate the abundance of biological factors. For example, homologous DNA recombination has been used extensively to effectively ablate protein-encoding genes to study protein function. Although this is still easiest in model organisms such as Saccharomyces cerevisiae that support extremely efficient homologous recombination, the availability of multiple genome sequences and technological advances that improve gene targeting, ${ }^{[14]}$ make this approach increasingly feasible in higher eukaryotes. In cases where gene deletion is not appropriate, for example in the study of essential proteins, the functions of these proteins can by queried by swapping endogenous gene promoters with an experimentally tunable promoter. Alternatively, RNA interference can be used to 'knock-down' expression of a protein of interest. ${ }^{[15]}$ The biggest strength of classical genetics is the fact that essentially any biological factor can be targeted; its biggest weakness is the time it takes to manipulate the system before experimentation can be performed. For example, classical genetic approaches are not suited to interrogate processes that occur on millisecond or even hour timescales. Additionally, classical genetic approaches are not well suited to study biological factors that can be compensated by other factors, or to study processes that occur with discrete spatial and/or temporal resolution. Tunable expression systems and RNA interference have similar and 
additional deficiencies. Again, the time required to attain the conditional inhibition is, biologically speaking, very long. Additionally, the perturbations required to install the conditional inhibition (e.g. temperature shift, carbon source shift, addition of siRNAs etc.) themselves often elicit 'off-target' effects that complicate interpretations of results. In contrast to classical genetics, chemical intervention can act within very short time frames, and, if highly specific for the biological factor of interest, do not elicit off-target side effects. However, unlike classical genetics that, in principle, can target any biological factor, at present it is exceeding difficult, costly and time consuming to identify small, cell-permeable molecules that specifically target a given biological factor. In some cases, combined chemicalgenetic approaches have been developed to genetically modify a biological factor rendering its activity susceptible to inhibition with a small, cell-permeable molecule. The Shokat group elegantly pioneered this approach with their chemicalgenetic inhibition of protein kinases. ${ }^{[16]}$ Unfortunately, even in the relatively welldeveloped field of protein kinase inhibition, this chemical genetic approach is still labor intensive and it works for only a subset of protein kinases. Clearly there is a great need for a generic, efficient, cost-effective method to discover cellpermeable molecules that can inhibit biological factors with high specificity and potency. Fulfilling this need is one of the goals of the NCCR Chemical Biology. Such chemical reagents would have exceptional value as tool compounds for fundamental molecular biology research, and, in many cases, these small molecules could serve as leads for eventual clinical applications.

\section{Sphingolipid Functions}

Ceramides and sphingolipids have been proposed to be important molecules both in signaling pathways and in membrane domain formation. However, it is difficult to intervene in ceramide biosynthesis in mammalian cells because there are six different ceramide synthases, which each have their own specificity with respect to the length of the fatty acyl coenzyme A they use as a substrate. Precedence for a role of specific ceramide synthases in physiology comes from experiments with the worm, C. elegans. Mutations in one ceramide synthase, hyl-2, caused a hypersensitivity to anoxic conditions, whereas the mutation in another ceramide synthase, hyl-1, caused an increased resistance to anoxia. The resistance to anoxia correlated with the ability of the worms to synthesize a class of ceramides and sphingomyelins with acyl chains of 20-22 carbons.[17] Recent studies from our lab suggest that other products of ceramide synthases might be critical in determining the sensitivity to anoxic conditions.

The only inhibitors that exist against ceramide synthases seem to act rather broadly, therefore it would be beneficial to have inhibitors that are specific to the individual enzymes. As ceramide synthases are membrane proteins, which are difficult to purify in large quantities, we will design a cellular assay that should allow us to identify specific inhibitors using the screening platform of the NCCR Chemical Biology, ACCESS. We have replaced the yeast ceramide synthases (LAG1 and $L A C 1$, see Fig. 1) with ceramide synthases from mammalian cells and we and others have shown that the enzymes retain their specificity in yeast.[18] This should allow us to develop high throughput comparative screening methods to identify compounds that specifically affect growth of the yeast housing mammalian ceramide synthases. Yeast have important advantages over mammalian cells in this type of screening. Besides the genetic manipulability and the robust growth yeast can be grown in defined medium, without addition of any lipid components. Growth medium for mammalian cells is much more complex and studies of lipids complicated by their presence in the growth medium. These experiments should also allow us to profile which sphingolipid changes in yeast modify responses to chemicals that inhibit yeast growth. We have previously shown that changes in lipid composition in cells can affect the sensitivity of yeast cells to compounds, such as rapamycin and caffeine. ${ }^{[19]}$

Ceramide synthases clearly have specificity towards the fatty acyl chain substrate. However, little is known about specificity towards the sphingoid base. Interestingly, recent studies have shown that deoxysphinganinesareimplicatedinhumandiseases. ${ }^{[20]}$ Mutations in the serine palmitoyltransferase increase its natural ability to accept alanine and glycine instead of serine. ${ }^{[21]}$ The resulting products lead to sphingoid bases that can be used to make deoxy- and deoxy, demethyl-ceramides. These are dead-end products with yet unknown physiological properties. We have developed techniques to synthesize various sphingoid bases and to test the ceramide synthases for specificity towards these precursors. These experiments should also enable us to explore the function of sphingoid bases and ceramides in physiology and determine the functions of the deoxysphingoid bases or deoxyceramides in cell biology and disease.

\section{Visualizing Physical Properties of Membranes}

The current methodology to report on the order parameter of membranes rely on fluorescent dyes using fluorescence anisotropy measurements ${ }^{[22]}$ or the analysis of the emission spectrum of dyes that distribute evenly in the membrane, but change their excitation/emission spectra depending upon membrane order.[23] The former technique has only been used to obtain average measurements over large areas of membrane, which is not very informative now that we know that membrane heterogeneity exists on a nanometer scale. The latter method, using dyes based on Laurdan, have the potential to be coupled with high resolution microscopy, but this is hampered by the properties of Laurdan and its detection by two-photon microscopy. A few dyes exist that are able to partition into membrane disordered or ordered domains in giant unilamellar vesicles (GUV), but their properties do not seem to be easily adaptable to studies on cells. Therefore, there is a need for the development of new fluorescent reporters and new technologies to examine membrane environments. We have prepared a series of fluorescent dyes, based on robust hydrophobic fluorescent compounds, naphthalenediimides (NDIs) and perylenediimides (PDIs) (see article by Montenegro et al. ${ }^{[24]}$. The excitation/emission properties of this set of compounds allow us to cover a very wide range of the visual spectrum. As the fluorescent dyes sit in the membrane environment their excitation/emission properties might change with the membrane environment. At the very least, their fluorescence lifetime should be proportional to the amount of water in the membrane, which correlates with membrane order. To render specific properties to the NDIs and PDIs, we perform simple reactions attaching various head groups and tails. These can be attached in various combinations leading to a large number of different compounds to test. The test we start with is to look for partitioning into liquid ordered or disordered membrane phases. We made GUVs with the following lipid composition in mole \%, dioleoylphosphatidylcholine/sphingomyelin(egg)/cholesterol $(0.24 / 0.56 / 0.2)$. This leads to vesicles that show separation into liquid ordered and disordered phases at room temperature. Fig. 2 shows a representative result obtained with one of the first novel dyes based on NDI. The new NDI dye in red is localized in liquid disordered phases, whereas the control dye, napthopyrene, in blue, is localized in liquid ordered phases. By changing the composition of the head and tail groups we expect to be able to identify novel dyes that partition into liquid ordered domains 


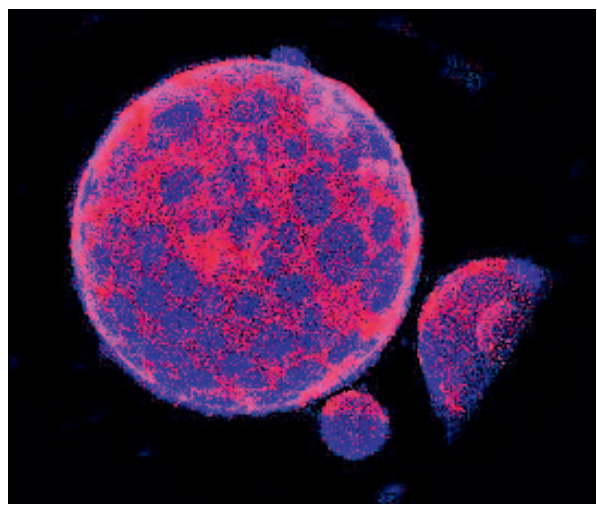

Fig. 2. Visualizing membrane microdomains. Giant unilamellar vesicles were grown using the lipid combinations described in the text. The blue dye, napthopyrene labels the liquid ordered phase of the GUVs. ${ }^{[25]}$ The novel, red, NDI-based fluorophore, labels the liquid disordered phase. This technique can be used to screen for the partitioning characteristics of novel fluorophores.

as well. It will also be interesting to examine the spectral properties of the dyes that do not partition to procure new compounds that can give quantitative readouts on the order of the membrane in which they sit. This type of compounds will be very useful in the following approach.

Above we have used the properties of dyes to partition in the membrane to reveal and measure membrane microdomains, but we are also developing novel techniques to examine the membrane environment surrounding membrane proteins. As a test case, we want to develop methods to report on the environment surrounding a signaling receptor and the changes that the receptor environment undergoes during signaling and endocytosis. We have created various chimeras where the SNAP tag[26] has been introduced into various locations of the insulin receptor. Our data show that several of these SNAP tagged receptors are signaling competent and seem to be downregulated normally by endocytosis. While the structure of the extracellular domain of the insulin receptor has been determined, ${ }^{[27]}$ the precise configuration of the juxtamembrane domain is not known in atomic detail. Therefore, we have made several constructs that should lead to a SNAP tag positioned relatively close to the external face of the membrane. Using benzylguanine-derivatized dyes, such as C-Laurdan or one of our new hydrophobic NDI or PDI based dyes, together with an appropriate sized linker we plan to attach the reporter dye directly to the receptor and have it partition into the lipid bilayer. In this way we localize the dye to the membrane immediately proximal to the receptor and we should be able to monitor the membrane properties in real-time. For reasons of sensitivity, we might not be able to monitor single receptors, but we can synchronize the system by coordinating the timing of the labeling of the SNAP tag with the application of the hormone. In this way, we should be able to determine if there are changes in the immediate membrane environment of the insulin receptor during signaling and endocytosis. Current results for this project look encouraging, but the technology is applicable to other cell surface molecules and we will perform similar studies on other molecules.

In summary, combination of chemical, physical and biochemical approaches will be used to obtain new insights into membrane structure and heterogeneity at a molecular level.

Received: September 28, 2011

[1] K. Simons, J. L. Sampaio, Cold Spring Harb. Perspect. Biol. 2011, 3, a004697.

[2] a) F. A. Heberle, G. W. Feigenson, Cold Spring Harb. Perspect. Biol. 2011, 3, a004630; b) I. Vattulainen, T. Rog, Cold Spring Harb. Perspect. Biol. 2011, 3, a004655.

[3] a) R. Varma, S. Mayor, Nature 1998, 394, 798; b) T. Friedrichson, T. V. Kurzchalia, Nature 1998, 394, 802

[4] D. Goswami, K. Gowrishankar, S. Bilgrami, S. Ghosh, R. Raghupathy, R. Chadda, R. Vishwakarma, M. Rao, S. Mayor, Cell 2008, $135,1085$.

[5] P. Sharma, R. Varma, R. C. Sarasij, Ira, K Gousset, G. Krishnamoorthy, M. Rao, S. Mayor, Cell 2004, 116, 577.

[6] a) T. Fujiwara, K. Ritchie, H. Murakoshi, K. Jacobson, A. Kusumi, J. Cell Biol. 2002, 157 , 1071; b) M. Tomishige, A. Kusumi, Mol. Biol. Cell 1999, 10, 2475.

[7] C. Eggeling, C. Ringemann, R. Medda, G. Schwarzmann, K. Sandhoff, S. Polyakova, V. N. Belov, B. Hein, C. von Middendorff, A. Schonle, S. W. Hell, Nature 2009, 457, 1159.

[8] R. G. Anderson, K. Jacobson, Science 2002, 296, 1821.

[9] P. S. Niemela, M. S. Miettinen, L. Monticelli, H. Hammaren, P. Bjelkmar, T. Murtola, E. Lindahl, I. Vattulainen, J. Am. Chem. Soc. 2010, 132,7574 .

[10] H. Ewers, A. Helenius, Cold Spring Harb. Perspect. Biol. 2011, 3.

[11] A. Yamaji, Y. Sekizawa, K. Emoto, H. Sakuraba, K. Inoue, H. Kobayashi, M. Umeda, J. Biol. Chem. 1998, 273, 5300.
[12] C. Heinis, T. Rutherford, S. Freund, G. Winter, Nat. Chem. Biol. 2009, 5, 502.

[13] K. M. Specht, K. M. Shokat, Curr. Opin. Cell. Biol. 2002, 14, 155 .

[14] M. Bibikova, K. Beumer, J. K. Trautman, D. Carroll, Science 2003, 300, 764.

[15] D. Grimm, Adv. Drug Deliv. Rev. 2009, 61, 672.

[16] A. C. Bishop, O. Buzko, K. M. Shokat, Trends Cell Biol. 2001, 11, 167.

[17] V. Menuz, K. S. Howell, S. Gentina, S. Epstein, I. Riezman, M. Fornallaz-Mulhauser, M. O. Hengartner, M. Gomez, H. Riezman, J. C. Martinou, Science 2009, 324, 381.

[18] I. Guillas, J. C. Jiang, C. Vionnet, C. Roubaty, D. Uldry, R. Chuard, J. Wang, S. M. Jazwinski, A. Conzelmann, J. Biol. Chem. 2003, 278, 37083.

[19] X. L. Guan, C. M. Souza, H. Pichler, G Dewhurst, O. Schaad, K. Kajiwara, H. Wakabayashi, T. Ivanova, G. A. Castillon, M. Piccolis, F. Abe, R. Loewith, K. Funato, M. R. Wenk, H. Riezman, Mol. Biol. Cell 2009, 20 , 2083.

[20] A. Penno, M. M. Reilly, H. Houlden, M. Laura, K. Rentsch, V. Niederkofler, E. T. Stoeckli, G. Nicholson, F. Eichler, R. H. Brown, Jr., A. von Eckardstein, T. Hornemann, J. Biol. Chem. 2010, 285, 11178.

[21] a) A. Rotthier, A. Penno, B. Rautenstrauss, M. Auer-Grumbach, G. M. Stettner, B. Asselbergh, K. Van Hoof, H. Sticht, N. Levy, V. Timmerman, T. Hornemann, K. Janssens, Hum. Mutat. 2011, 32, E2211; b) A. Rotthier, M. Auer-Grumbach, K. Janssens, J. Baets, A. Penno, L. AlmeidaSouza, K. Van Hoof, A. Jacobs, E. De Vriendt, B. Schlotter-Weigel, W. Loscher, P. Vondracek, P. Seeman, P. De Jonghe, P. Van Dijck, A Jordanova, T. Hornemann, V. Timmerman, Am. J. Hum. Genet. 2010, 87, 513 .

[22] B. R. Lentz, Chem. Phys. Lipids 1993, 64, 99.

[23] H. M. Kim, H. J. Choo, S. Y. Jung, Y. G. Ko, W. H. Park, S. J. Jeon, C. H. Kim, T. Joo, B. R. Cho, Chembiochem 2007, 8, 553.

[24] J. Montenegro, C. Gehin, E.-K. Bang, A. Fin, D. Alonso Doval, H. Riezman, N. Sakai, S. Matile, Chimia 2011, 65, 853.

[25] N. F. Morales-Penningston, J. Wu, E. R. Farkas, S. L. Goh, T. M. Konyakhina, J. Y. Zheng, W. W. Webb, G. W. Feigenson, Biochim. Biophys. Acta 2010, 1798, 1324.

[26] A. Keppler, S. Gendreizig, T. Gronemeyer, H Pick, H. Vogel, K. Johnsson, Nat. Biotechnol. 2003, 21,86 .

[27] N. M. McKern, M. C. Lawrence, V. A. Streltsov, M. Z. Lou, T. E. Adams, G. O. Lovrecz, T. C. Elleman, K. M. Richards, J. D. Bentley, P. A. Pilling, P. A. Hoyne, K. A. Cartledge, T. M. Pham, J. L. Lewis, S. E. Sankovich, V. Stoichevska, E. Da Silva, C. P. Robinson, M. J. Frenkel, L. G. Sparrow, R. T. Fernley, V. C. Epa, C. W. Ward, Nature 2006, 443, 218. 